\title{
Anel vascular: uma causa de desconforto respiratório neonatal frequentemente esquecida
}

\author{
Vascular ring: a commonly overlooked cause of neonatal respiratory distress
}

\author{
Ellen Binotto de Castro ${ }^{1}$, Guilherme Ricardo Nunes Silva ${ }^{1}$, \\ Nathalie Jeanne Magioli Bravo-Valenzuela ${ }^{2}$
}

Castro EB, Silva GRN, Bravo-Valenzuela NJM. Anel vascular: uma causa de desconforto respiratório neonatal frequentemente esquecida/ Vascular ring: a commonly overlooked cause of neonatal respiratory distress. Rev Med (São Paulo). 2015 jul.-set.;94(3):189-96.

RESUMO: Objetivo: Relatar o caso de um recém-nascido com anel vascular completo causado por artéria subclávia esquerda retroesofágica em arco aórtico à direita, associado a um divertículo de Kommerell. Relato de Caso: Paciente do gênero feminino, prematuro (36 semanas e 3 dias), baixo peso (1670g), nasceu de parto via cesária e com Apgar 7/8. Ao exame físico notava-se dispneia, cianose, estridor inspiratório e sopro sistólico (2/6) em borda esternal esquerda alta. A paciente foi levada para unidade de terapia intensiva neonatal com melhora imediata do padrão respiratório e da cianose após intubação orotraqueal. A radiografia de tórax demonstrou alargamento do mediastino e arco aórtico à direita. $\mathrm{O}$ ecocardiograma transtorácico evidenciou: forame oval pérvio, arco aórtico à direita e tortuoso, dilatação da aorta ascendente, canal arterial pérvio grande, shunt esquerda-direita pelo septo interatrial e canal arterial e sinais de obstrução ao fluxo da artéria pulmonar direita por provável compressão vascular. A angiotomografia confirmou o anel vascular e esclareceu sua anatomia: arco aórtico à direita com artéria subclávia esquerda retroesofágica originando-se de um divertículo (Kommerell) associada à um canal arterial patente à esquerda. A artéria subclávia esquerda anômala ocasionava compressão extrínseca esofagotraqueal, do fluxo da artéria pulmonar direita e do brônquio fonte à direita. À esquerda, o grande canal arterial patente completava o anel vascular, o que ocasionou sintomas precoces. Conclusões: $\mathrm{O}$ anel vascular é uma malformação congênita que deve ser suspeitada em todos os pacientes com anomalias do arco aórtico que apresentam sintomas respiratórios (dispneia/estridor inspiratório e/ou sibilância crônica).

Descritores: Artéria subclávia/anormalidades; Artéria subclávia/ radiografia; Recém-nascido; Síndrome do desconforto respiratório do récem-nascido; Síndrome do arco aórtico/radiografia; Imagem tridimensional; Feminino.

\begin{abstract}
Objective: To report a case of a newborn with complete vascular ring caused by the right-sided aortic arch with retroesophageal left subclavian artery and Kommerell diverticulum. Case Report: A preterm newborn female (36 weeks 3 days) with low birth weight ( $1670 \mathrm{~g}$ ) was delivered by cesarean section, with Apgar scores of 7/8. Physical examination revealed dyspnea, cyanosis, and inspiratory stridor with systolic murmur ( $2 / 6$ grade) at the left upper sternal border. She was transferred to the neonatal intensive care unit, with improvement in her cyanosis and respiratory distress, immediately after orotracheal intubation. Chest X-ray demonstrated mediastinal enlargement with a rightsided aortic arch. The transthoracic echocardiogram showed situs solitus, levocardia, foramen ovale, tortuous and right-sided aortic arch, enlarged ascending aorta, large patent ductus arteriosus (PDA), left-to-right shunt at atrial septal and PDA levels, and Doppler signs of right pulmonary artery flow obstruction probably caused by vascular compression. Angiotomography confirmed the vascular ring and detailed his obstruction anatomy: the rightsided aortic arch with the retroesophageal left subclavian artery arising from the Kommerell diverticulum and left-sided PDA. The anomalous left subclavian artery caused the tracheo-esophageal, right pulmonary artery, and right bronchus compression. The leftsided large PDA completed the vascular ring and its combination caused early symptoms. Conclusion: Vascular ring it's a congenital malformation that should be suspected in all patients associated with aortic arch anomaly presenting with respiratory symptoms (dyspnea/inspiratory stridor and/or chronic sibilance).
\end{abstract}

Keywords: Subclavian artery/abnormalities; Subclavian artery/ radiography; Infant, newborn; Respiratory distress syndrome, newborn; Aortic arch syndromes/radiography; Imaging, threedimensional; Female.

1. Acadêmicos de Medicina, Universidade de Taubaté, Taubaté, SP.E-mails: ellenbinotto@hotmail.com; guilherme.r.n.silva@gmail.com.

2. Doutora em Cardiologia Pediátrica, Professora de Pediatria da Universidade de Taubaté, Taubaté, SP. E-mail: nathaliejeanne@ pedicor.com.br.

Autor para correspondência: Nathalie Jeanne Magioli Bravo-Valenzuela. Universidade de Taubaté. Av. Tiradentes, $\mathrm{n}^{\circ} 500$. Bom $^{2}$ Conselho. Taubaté, SP. CEP: 12030-180. E-mail: nathaliejeanne@pedicor.com.br. 


\section{INTRODUÇÃO}

$\mathrm{O}$ s anéis vasculares são anomalias congênitas do arco aórtico e vasos da base com compressão extrínseca da traqueia, do esôfago, ou ambos e que podem manifestar-se com distúrbios respiratórios e/ ou digestivos ${ }^{1}$. Representam de $1-2 \%$ de todas as anomalias cardiovasculares congênitas, constituindo importante causa de obstrução traqueal ${ }^{2,3}$. Dentre os diversos tipos de anel vascular, classificados pelo International Congenital Heart Surgery Nomenclature and Database Commitee (Tabela 1), a artéria inominada direita, o duplo arco aórtico e o arco aórtico à direita são os mais comuns ${ }^{4,5}$. Podem ser completos, quando circundam toda a região traqueoesofágica, ou incompletos, quando comprimem apenas uma porção esofágica e/ou traqueal ${ }^{2,6}$.

Tabela 1 - Classificação dos anéis vasculares de acordo com o International Congenital Heart Surgery Nomenclature and Database Committee $e^{4,5}$

\begin{tabular}{l}
\hline Duplo arco aórtico \\
\hline Arco aórtico direito dominante \\
Arco aórtico esquerdo dominante \\
Arcos balanceados \\
\hline Arco aórtico direito - ligamento esquerdo \\
\hline Imagem em espelho dos ramos arteriais \\
Artéria subclávia esquerda retroesofágica \\
Aorta circunflexa \\
\hline Compressão da artéria inominada \\
\hline Compressão da artéria pulmonar
\end{tabular}

A presença dos sintomas e sua intensidade dependem do tipo e da localização do anel vascular, podendo variar desde intensos até inexistentes. Nos casos assintomáticos, o diagnóstico costuma ser um achado acidental. Quando presentes, os sintomas mais comuns são: estridor respiratório e sibilância crônica nos lactentes e nas crianças acima de um ano, a disfagia para sólidos. Entretanto, este diagnóstico pode ser difícil, devido sua possível confusão com outras doenças, principalmente as respiratórias ${ }^{1}$. O diagnóstico precoce, por vezes pré-natal, é importante, pois pode evitar o desenvolvimento de possíveis complicações, como a traqueomalácea ou lesões crônicas do parênquima pulmonar ${ }^{2,6}$.

Os anéis vasculares resultam do desenvolvimento embriológico anormal de segmentos específicos do arco aórtico primitivo ou rudimentar ${ }^{7}$. Normalmente, a porção direita do quarto arco aórtico primitivo regride e a porção esquerda persiste como arco aórtico esquerdo. Quando persiste toda a aorta dorsal e apenas a porção distal da esquerda resulta em arco aórtico à direita. Estes casos, quando isolados, são assintomáticos, estando a presença dos sintomas relacionados a possíveis anomalias congênitas associadas ou à compressão de estruturas do mediastino por vasos (anel vascular). Raramente pode ocorrer uma dilatação na origem do vaso aberrante chamada de aneurisma ou divertículo de Kommerell. O divertículo de Kommerell é mais comumente associado com arco aórtico à esquerda e artéria subclávia anômala (ASA) direita (0,5$2 \%$ ) do que com o arco aórtico à direita e ASA esquerda $(0,05-0,1 \%)^{8,9,10}$. Neste último, o arco aórtico à direita, a ASA e o canal arterial ou ligamento arterioso circundam a traqueia e esôfago, resultando em anel vascular completo, em que a dilatação do vaso anômalo (divertículo) reforça a compressão. Em geral, os sintomas destes pacientes com divertículo de Kommerell são tardios, manisfestando-se na idade adulta com disfagia, dor torácica. Entretanto, em alguns casos, os sintomas podem ocorrer nos primeiros meses de vida, sendo quadro respiratório mais frequente em lactentes ${ }^{6,11}$.

Neste estudo descrevemos um caso de anel vascular completo com divertículo de Kommerell em arco aórtico à direita, que ocasionou sintomas precoces (período neonatal) enfatizando a importância de se considerar este diagnóstico diferencial na apresentação clínica de sintomas respiratórios.

\section{RELATO DO CASO}

Recém-nascido do gênero feminino, pré-termo (Capurro: 36 semanas e 3 dias), muito baixo peso (1670g), pequeno para a idade gestacional, nasceu de parto Cesária com Apgar 7 e 8 ( $1^{\circ}$ e $5^{\circ}$ minutos). Diagnóstico pré-natal de restrição do crescimento intrauterino decorrente de doença hipertensiva crônica. Evoluiu com desconforto respiratório precoce e cianose generalizada sendo então realizada intubação orotraqueal, com melhora imediata do padrão respiratório e da cianose.

Ao exame físico, antes da intubação traqueal, notava-se taquidispneia, cianose e estridor inspiratório. Na propedêutica cardiológica, o ritmo cardíaco era regular em dois tempos, com sopro sistólico $2 / 6$ em borda esternal esquerda alta, os pulsos arteriais eram palpáveis, amplos e simétricos, e os valores de pressão arterial normais nos quatro membros. Na radiografia de tórax observouse alargamento do mediastino (Figura 1). Realizado ecocardiograma que evidenciou: situs solitus, levocardia; forame oval pérvio com shunt E/D; concordância atrioventricular e ventrículo-arterial; insuficiência tricúspide discreta; dilatação das cavidades esquerdas; arco aórtico à direita, tortuoso, com dilatação importante na porção ascendente (Figura 2A), canal arterial pérvio grande com fluxo direcionado da aorta para a artéria pulmonar, obstrução ao fluxo da artéria pulmonar direita (Figura 2B); difícil visualização da origem da artéria pulmonar esquerda; 
possível presença de circulação colateral no pulmão direito.

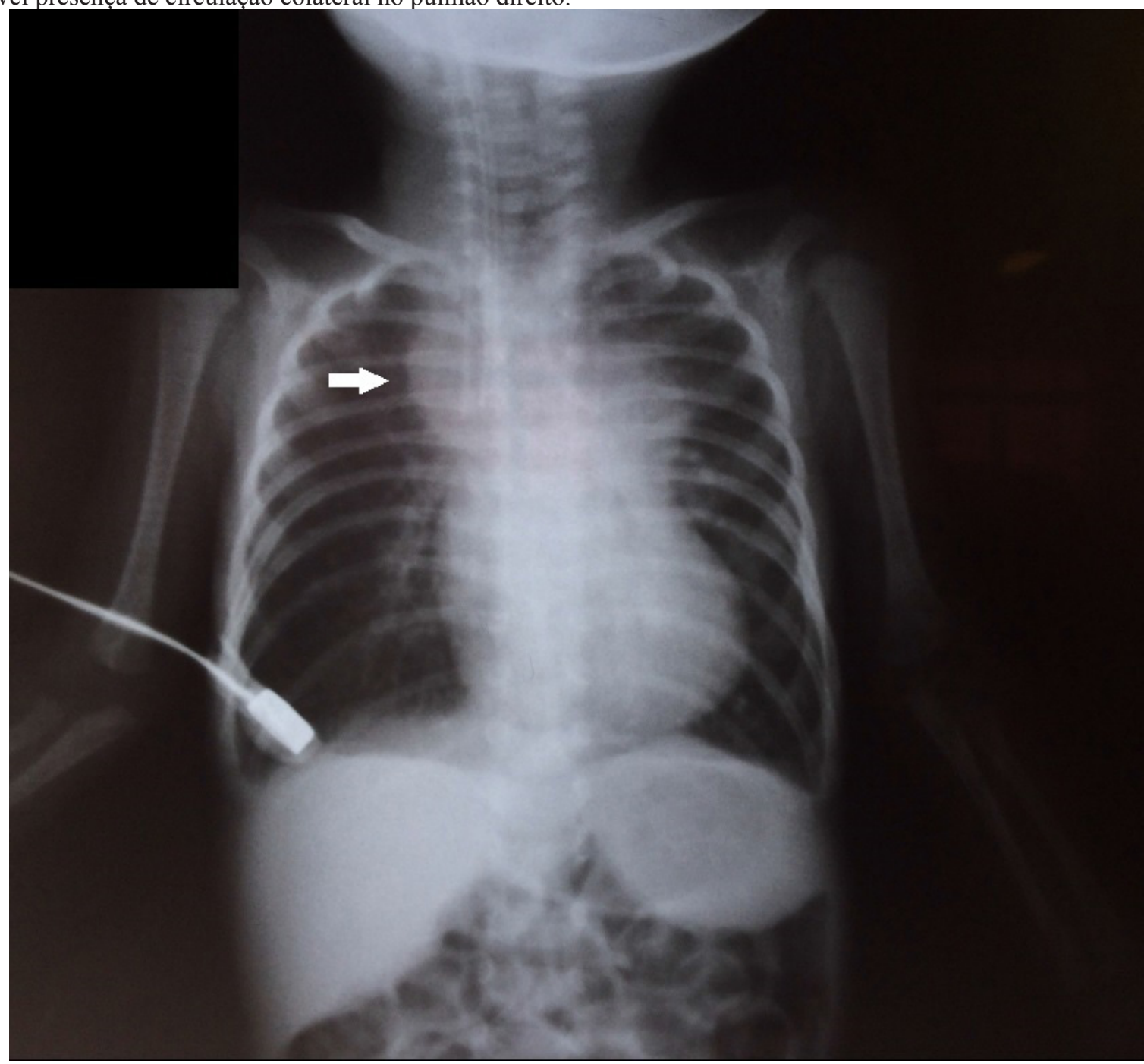

Figura 1 - Radiografia de tórax em anteroposterior demonstrando mediastino alargado e arco aórtico à direita (seta)
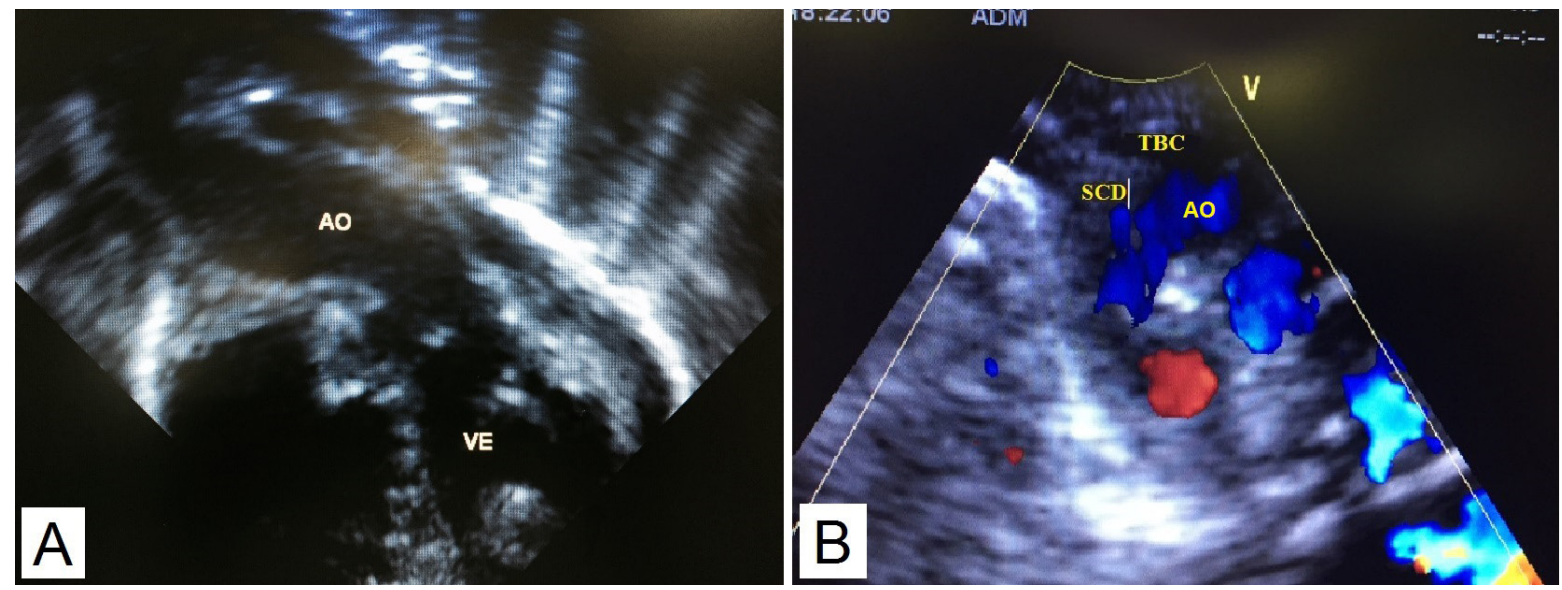

Figura 2 - Ecocardiograma transtorácico do caso: A) plano subcostal longo eixo demonstrando que a artéria aorta (AO) origina-se do ventrículo esquerdo (VE), é tortuosa e apresenta dilatação de sua porção ascendente, B) plano supraesternal longo eixo demonstrando arco aórtico (AO) à direita e dois vasos originando-se do arco: tronco braquiocefálico (TBC) esquerdo e em seguida a artéria subclávia direita $(\mathrm{SCD})$ 
Devido a suspeita clínica e ecocardiográfica de anel vascular, foi realizada angiotomografia, que evidenciou: artérias pulmonares (direita e esquerda) confluentes e com origem distante; estenose importante da artéria pulmonar direita por compressão extrínseca da artéria subclávia esquerda anômala e ausência de circulação colateral. O arco aórtico apresentava-se à direita da coluna com dilatação importante da aorta ascendente e origem anormal dos vasos supra-aórticos. Ambas as artérias carótidas (esquerda e direita) e a subclávia esquerda originavam-se de um divertículo do primeiro vaso que emergia da aorta (tronco braquiocefáico esquerdo) e o segundo vaso correspondia à artéria subclávia direita (Figuras 3A, 3B e 3C). A artéria subclávia esquerda apresentava trajeto anômalo (cranial e para a direita), comprimindo a artéria pulmonar direita, a traqueia e o esôfago (Figura 4). Da região ístmica, originava-se grande canal arterial, alongado, com trajeto retrotraqueal e retroesofágico, completando o anel vascular à esquerda (Figuras 5A e 5B). Como variação anatômica da árvore respiratória, observou-se presença de brônquio traqueal, responsável pela ventilação do lobo superior do pulmão direito, e estenose discreta do brônquio fonte direito (Figura 6).

Depois de confirmado e esclarecido o diagnóstico da anatomia do anel vascular, foi indicado o tratamento cirúrgico para ligadura e secção da ASA esquerda e do canal arterial, por tratar-se de uma criança sintomática. Entretanto, o recém-nascido evoluiu com quadro de hemorragia digestiva alta, insuficiência renal aguda, sepse fúngica com endocardite (vegetações no ventrículo direito e esquerdo) e choque, falecendo no $55^{\circ}$ de internação, antes da correção cirúrgica da cardiopatia.

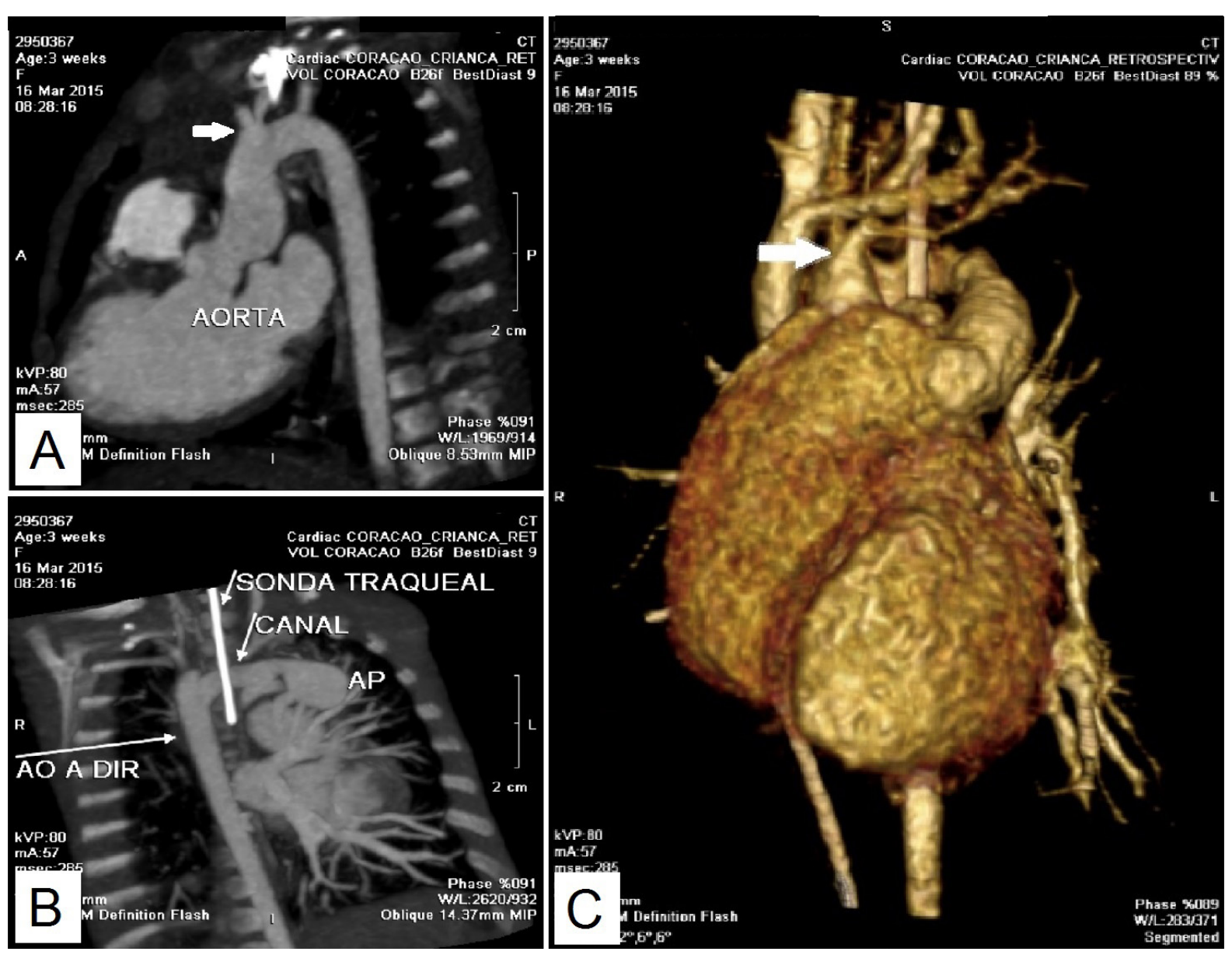

Figura 3 - Angiotomografia do caso com imagens em 3D: A) aorta ascendente tortuosa e dilatada, tronco braquiocefálico esquerdo com divertículo de onde origina-se a artéria subclávia esquerda e aorta descendente à direita da coluna (seta), B) reconstrução tridimensional da artéria subclávia esquerda (seta) com origem anômala, C) plano longitudinal demonstrando aorta (AO) descendo à direita da coluna, com anel vascular e artéria subclávia esquerda à direita da traqueia (SONDA TRAQUEAL) e canal arterial (CANAL) completando o anel vascular à esquerda com a artéria pulmonar (AP) 


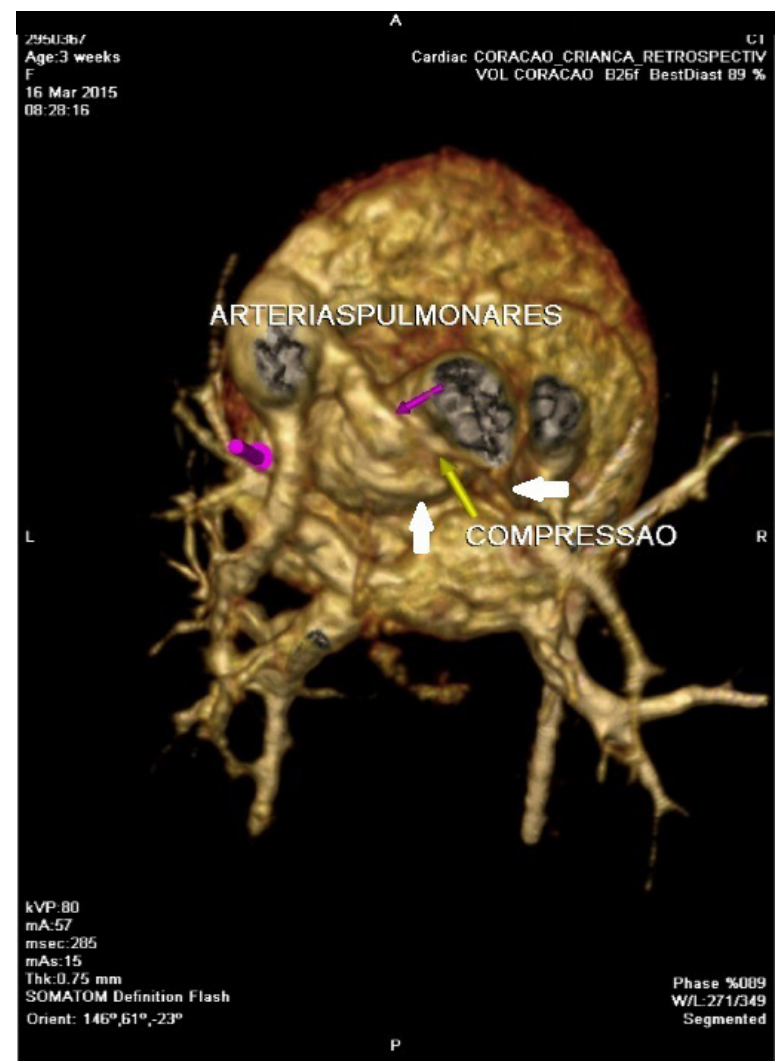

Figura 4 - Angiotomografia do caso com imagens em 3D: artéria subclávia esquerda (setas brancas) comprimindo a artéria pulmonar direita

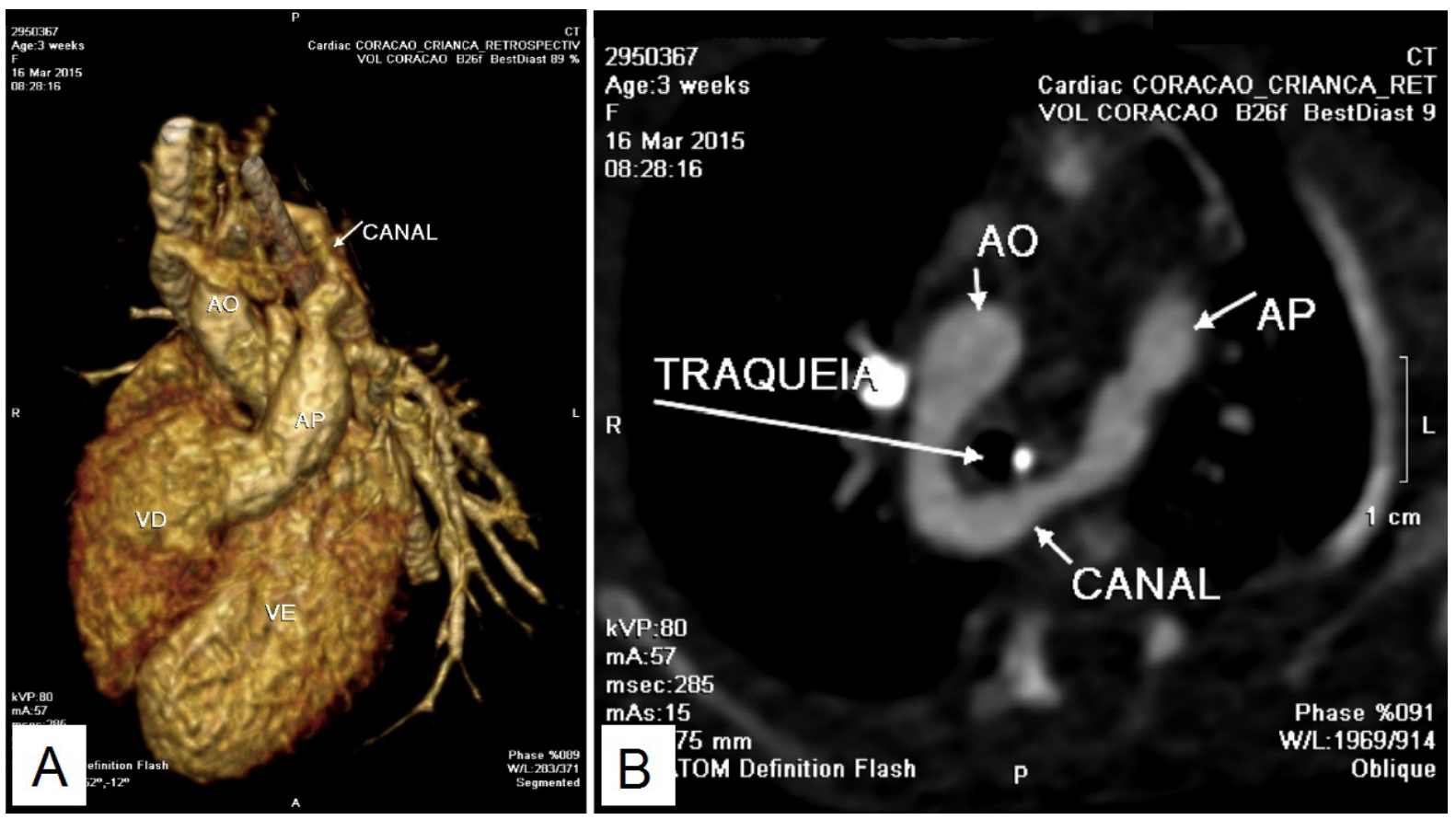

Figura 5 - A) Imagem em 3D da angiotomografia, visão anterior demonstrando grande canal arterial com trajeto retroesofágico e retrotraqueal e B) Tomografia computadorizada do paciente demonstrando, no plano transverso do mediastino superior, o anel vascular (forma de U) em torno da traqueia. Aorta (AO), artéria pulmonar (AP), ventrículo direito (VD), ventrículo esquerdo (VE), canal arterial (CANAL) e traqueia (TRAQUEIA) 


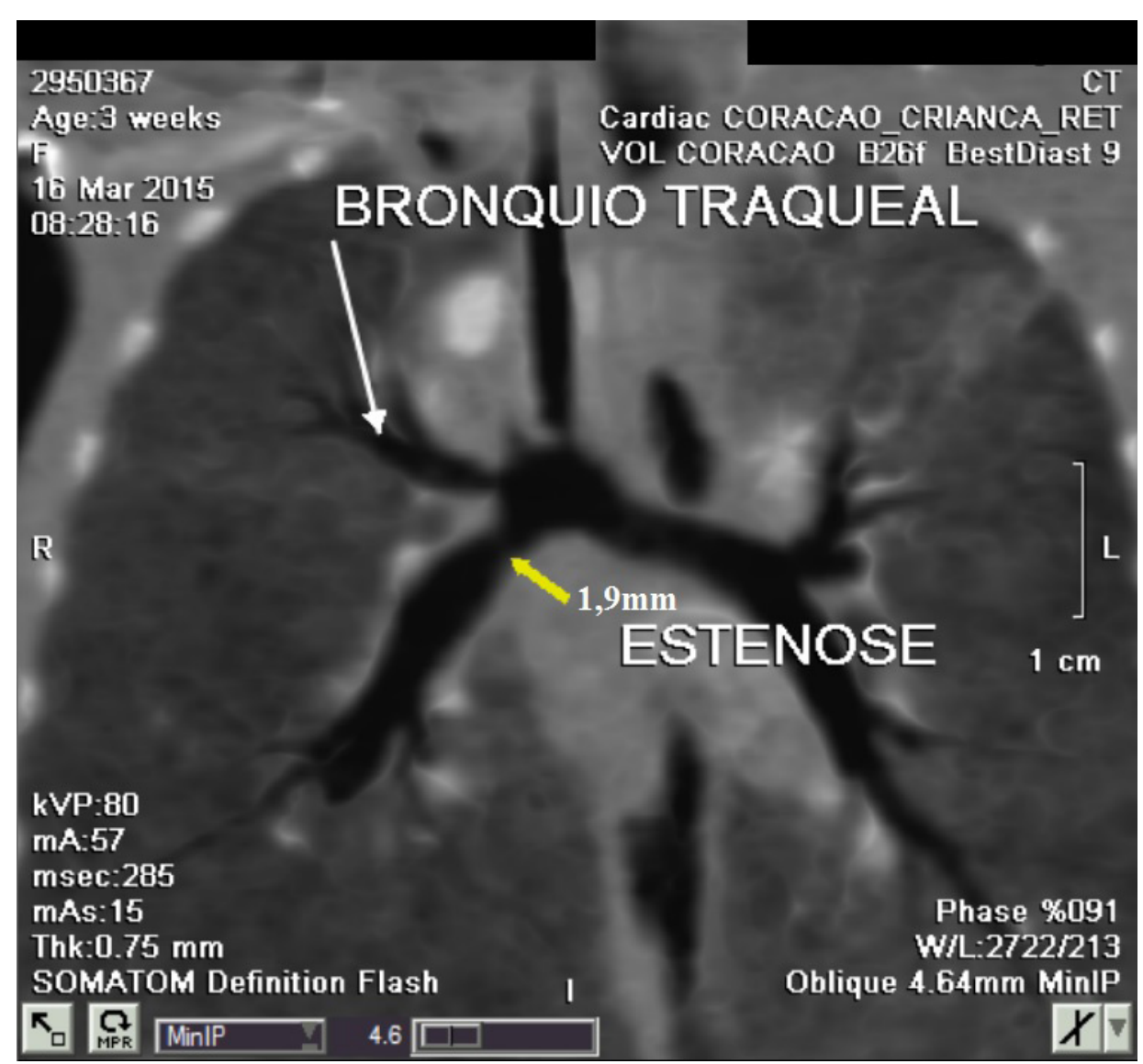

Figura 6 - Imagem de angiotomografia em corte coronal com presença de brônquio traqueal à direita e estenose importante do brônquio fonte direito $(1,9 \mathrm{~mm})$

Depois de confirmado e esclarecido o diagnóstico da anatomia do anel vascular, foi indicado o tratamento cirúrgico para ligadura e secção da ASA esquerda e do canal arterial, por tratar-se de uma criança sintomática. Entretanto, o recém-nascido evoluiu com quadro de hemorragia digestiva alta, insuficiência renal aguda, sepse fúngica com endocardite (vegetações no ventrículo direito e esquerdo) e choque, falecendo no $55^{\circ}$ de internação, antes da correção cirúrgica da cardiopatia.

\section{DISCUSSÃO}

Os anéis vasculares que resultam em compressão sintomática das vias aéreas são malformações raras $(0,8$ $1,3 \%)^{2,3}$. Os sintomas respiratórios podem variar desde cianose exacerbada por choro ou pela alimentação, até estridor respiratório ou mesmo um quadro de sibilância de difícil controle ${ }^{2,6}$. A intensidade e precocidade das manifestações clínicas dependem da tensão exercida pelo anel sobre as vias respiratórias, podendo ter início, no período neonatal ou mais tardiamente. Considerando-se as características anatômicas e funcionais das vias aéreas de recém-nascidos e lactentes: menor calibre traqueal (cerca de 3-6 mm) e fluxo aéreo não laminar, qualquer redução do diâmetro interno do trato respiratório ocasiona importante repercussão na dinâmica respiratória nesta faixa etária. Como os sintomas, em geral, são diversos e inespecíficos, podem ser confundidos com doenças respiratórias, dificultando a suspeita da compressão traqueal, tornando o tratamento mais tardio, o que aumenta a morbidade. Desta forma, o diagnóstico de anel vascular, embora raro, deve fazer parte das possíveis etiologias a serem consideradas pelo pediatra na sequencia propedêutica de um "bebê chiador".

A artéria subclávia anômala (ASA) é uma das mais comuns anomalias congênitas do arco aórtico, sendo mais rara a ASA esquerda $(0,05-0,1 \%)$ que a direita $(0,5-$ $2,0 \%)^{6,11}$. Em aproximadamente $60 \%$ dos casos de ASA existe uma dilatação cônica ou divertículo na origem do vaso aberrante ${ }^{12}$. Estes divertículos podem ser de três tipos: tipo I - divertículo em arco aórtico à esquerda com artéria subclávia direita anômala, tipo II - divertículo em arco aórtico à direita com artéria subclávia esquerda anômala e tipo III - divertículo aórtico em junção aortoductal ${ }^{13}$. O primeiro tipo foi originalmente descrito por Kommerell, em um caso de subclávia direita aberrante com arco aórtico à esquerda, sendo a combinação de subclávia esquerda aberrante em arco aórtico à direita extremamente rara9 
Relatamos neste estudo um caso de arco aórtico dextroposto com subclávia esquerda aberrante ocasionando compressão da artéria pulmonar direita e da traqueia (anel incompleto), com presença de grande canal arterial à esquerda, completando o anel vascular (anel completo), comprimindo a traqueia e também o esôfago (Figuras 4, 5A e 5B).

O principal diagnóstico diferencial do divertículo de Kommerell é com anel vascular do tipo duplo arco aórtico. Este último resulta do não desaparecimento embriológico da porção distal da aorta dorsal direita e persistência de dois arcos aórticos (direito e esquerdo). As manifestações clínicas destas malformações são: estridor, infecções respiratórias recorrentes, e tosse crônica, frequentemente associada à disfagia aos sólidos, e costumam ser mais tardias no divertículo de Kommerell que no duplo arco aórtico ${ }^{6,11}$. Entretanto, no presente caso, apesar da manifestação clínica precoce (período neonatal) tratava-se de um divertículo de Kommerell. Nas crianças, os sintomas respiratórios são mais comuns, enquanto que nos adultos predominam a disfagia e dor torácica. Alguns pacientes com divertículo de Kommerell são assintomáticos até a idade adulta e como o divertículo apresenta risco elevado de dissecção ou ruptura, os sintomas iniciais da doença podem advir da dissecção ou dilatação aneurismática ${ }^{11}$. Outras causas de compressão não vascular do esôfago e da traqueia devem ser consideradas no diagnóstico diferencial dos anéis vasculares: massas mediastinais, cistos broncogênicos e cistos de duplicação esofágica $^{1,8}$.

Na suspeita do anel vascular, o esofagograma é o exame utilizado na abordagem inicial. Ele demonstra a presença da compressão posterior do esôfago presente em todos os anéis vasculares, exceto no pulmonar sling em que a compressão do esôfago é anterior e na artéria inominada, em que o esofagograma é normal ${ }^{14}$. A traqueobroncoscopia é um método diagnóstico muito útil na investigação destas malformações vasculares, pois permite a visualização da compressão pulsátil anormal na traqueia e o diagnóstico de traqueomalácia, comumente associada nestes $\operatorname{casos}^{14}$. Em especial nas crianças em ventilação mecânica, pela dificuldade de realização do esofagograma, pode ser o primeiro exame a ser solicitado. O ecocardiograma afasta outras malformações cardíacas e permite a suspeita de anel vascular, já no período pré-natal (ecocardiograma fetal) pela lateralidade do arco aórtico e emergência anômala de $\operatorname{vasos}^{15}$. Nos últimos quinze anos, com o avanço das técnicas com reconstrução de imagens tridimensionais

\section{REFERÊNCIAS}

1. Longo-Santos LR, Maksoud-Filho JG, Tannuri U, Andrade WC, Gonçalves MEP, Cardoso SR, et al. Anéis vasculares na infância: diagnóstico e tratamento. J Pediatr. 2002;78(3):24450. Disponível em: http://www.scielo.br/pdf/jped/v78n3/ v78n3a14.pdf.
(3D), como a angiografia por tomografia computadorizada (angio-TC) e a ressonância nuclear magnética (angioRM) do coração, vem sendo utilizadas para confirmar o diagnóstico do anel vascular. A arteriografia percutânea (cateterismo cardíaco) apesar de possibilitar o diagnóstico anatômico de certeza, deve ser evitada, por ser um método invasivo, sobretudo no período neonatal pela morbidade elevada em recém-nascidos de baixo peso ${ }^{16}$. Desta forma a angio-TC e a angio-RM, por permitirem a avaliação anatômica da malformação vascular e afastarem possíveis malformações respiratórias associadas, vem substituindo o uso do cateterismo cardíaco no diagnóstico e planejamento terapêutico dos anéis vasculares ${ }^{17}$. No presente relato, o recém-nascido apresentava baixo peso $(1600 \mathrm{~g})$, o que inviabilizou a realização da fibroscopia traqueal no nosso serviço (fibroscópio para recém-nascido não disponível), optando-se pela realização da angio-TC devido ao risco aumentado para o cateterismo cardíaco ${ }^{16}$.

$\mathrm{Na}$ maioria dos casos de ASA, a via de acesso cirúrgico é por toracotomia póstero-lateral esquerda, em que se faz a ligadura e a secção da ASA direita, originária da aorta descendente, cruzando posteriormente o esôfago, entre o mesmo e a coluna. Com exceção do anel da artéria pulmonar (pulmonar sling), não é necessária circulação extracorpórea para a correção destas anomalias. $\mathrm{Na}$ presença de divertículo de Kommerell, recomenda-se o reparo do divertículo, secção e sutura do ligamento arterioso ou do canal arterial, e o reimplante da artéria subclávia na artéria carótida esquerda. Nos adultos, vem sendo associado à cirurgia o procedimento endovascular (híbrido ${ }^{18}$. A complicação operatória mais frequente é o quilotórax, recomendando-se sempre a dissecção próxima ao divertículo ${ }^{(19)}$. Outras complicações no pósoperatório são a persistência dos sintomas respiratórios, frequentemente decorrentes da traqueomalácea, da estenose traqueal ou de lesões do parênquima pulmonar.

\section{CONCLUSÃO}

O anel vascular deve ser suspeitado em crianças com sintomas respiratórios de início no período neonatal e com sibilância persistente ("bebê chiador"). O diagnóstico precoce possibilita o tratamento, reduzindo as complicações pós-operatórias que comprometem a dinâmica e estrutura traqueais (como a traqueomalácea e a estenose da traquéia) e lesões crônicas do parênquima pulmonar, que comprometem a funcionalidade das vias aéreas.

2. Hernanz-Schulman M. Vascular rings: a practical approach to imaging diagnosis. Pediatr Radiol. 2005;35:961-79. doi: 10.1007/s00247-005-1529-0.

3. Simões AS, Peres A, Valeste S, et al. Duplo arco aórtico: causa rara de estridor. Acta Pediatr Portug. 2009;40(6):268-70. 
Disponível em: http://goo.gl/0Au7sO.

4. Backer CL, Mavroudis C. Congenital Heart Surgery Nomenclature and Database Project: vascular rings, tracheal stenosis, pectus excavatum. Ann Thorac Surg. 2000;69(4 Suppl):S308-18. doi: 10.1016/S0003-4975(99)01279-5.

5. Abrão AR, Souza VQ, Campos EO, Brito RK, Mançano AD. Duplo arco aórtico: a quebra do silêncio. J Vasc Bras. 2011;10(1):59-63. http://dx.doi.org/10.1590/S167754492011000100011 .

6. Lowe GM, Donaldson JS, Backer CL. Vascular rings: 10year review of imaging. RadioGraphics. 1991;11:637-46. DOI: http://dx.doi.org/10.1148/radiographics.11.4.1887119.

7. Abuchaim DCS, Burger M, Berwanger SA, Faraco DL. Anel vascular associado a divertículo de Kommerell: relato de caso. Rev Bras Cir Cardiovasc. 2007;22(3):352-4. http://dx.doi. org/10.1590/S0102-76382007000300014.

8. Alves EV, Valente RBM, Lucas E, Almeida RM, Soffe BA, Teldeshi AL, et al. Anel vascular: uma causa rara de estridor na infância. Revista de Pediatria SOPERJ. 2006;7(1):15-9. file://C:/Users/Administrador/Downloads/v7n1a04\%20(1). pdf.

9. Barranhas AD, Indiani JMC, Marchiori E, Santos AASMD, Rochitte CE, Nacif MS. Apresentação atípica de divertículo de Kommerell. Arq Bras Cardiol. 2009;93(6):e101-3. http:// dx.doi.org/10.1590/S0066-782X2009001200026.

10. Faucz RA, Furlan S, Barros AS, Bof AM, Lomonte ES, Leiro LC, et al. Arco aórtico direito com artéria subclávia esquerda aberrante e divertículo de Kommerell. Radiol Bras. 2005;38(5):381-4. http://dx.doi.org/10.1590/S010039842005000500014 .

11. Ota T, Okada K, Takanashi S, Yamamoto S, Okita Y. Surgical treatment for Kommerell's diverticulum. J Thorac Cardiovasc
Surg 2006;131(3):574-8. http://dx.doi.org/10.1016/j. jtcvs.2005.10.012.

12. Kadir S. Regional anatomy of the thoracic aorta. In: Kadir $\mathrm{S}$, editor. Atlas of normal and variant angiographic anatomy. Philadelphia: WB Saunders; 1991. p.19-54.

13. Salomonowitz E. The three types of aortic diverticula. AJR Am J Roentgenol. 1984;142:673-9. http://www.ajronline.org/ doi/pdf/10.2214/ajr.142.4.673.

14. Backer CL, Mavroudis C, Rigsby CK, Holinger LD. Trends in vascular ring surgery. J Thorac Cardiovasc Surg. 2005;129(6):1339-47. http://dx.doi.org/10.1016/j. jtcvs.2004.10.044.

15. Backes CH, Cua C, Kreutzer J, Armsby L, El-Said H, Moore $\mathrm{JW}$, et al. Low weight as an independent risk factor for adverse events during cardiac catheterization of infants. Catheter Cardiovasc Interv. 2013;82:786-94. doi: 10.1002/ccd.24726.

16. Pedra SRFF, Camillotti LP, Ishikawa WY, Martins LM, Assef JE, Sousa AGMR. Diagnóstico pré-natal de arco aórtico para a direita e origem aberrante da artéria subclávia esquerda. Rev Bras Ecocardiogr Imagem Cardiovasc. 2011;24(1):107-11. Disponível em: http://goo.gl/nGfm2z.

17.Etesami M, Ashwath R, Kanne J, Gilkeson RC, Rajiah P. Computed tomography in the evaluation of vascular rings and slings. Insights Imaging. 2014;5:507-21. doi: 10.1007/ s13244-014-0343-3.

18. Yang C, Shu C, Li M, Li Q, Kopp R. Aberrant subclavian artery pathologies and Kommerell's diverticulum: a review and analysis of published endovascular/hybrid treatment options. J Endovasc Ther. 2012;19(3):373-82.

19. Tanaka A, Milner R, Ota T. Kommerell's diverticulum in the current era: a comprehensive review. Gen Thorac Cardiovasc Surg. 2015;63(5):245-59. doi: 10.1007/s11748-015-0521-3. 